\title{
Disambiguating cues of disjunctive questions
}

\author{
Mohammad Ali. S. Bani Younes, Sam Hellmuth \\ Language and Linguistic Science, University of York, UK \\ https://doi.org/10.36505/ExLing-2020/11/0010/000425
}

\begin{abstract}
This paper aims to find out the similarities and differences between Jordanian (JA), Egyptian (EA), and Kuwaiti (KA) Arabic in which cues disambiguate alternative questions (altqs) and disjunctive yes-no questions (dynqs): intonation contour and choice of disjunctive element (DE). A perception study was run in the three dialects, replicating Pruitt \& Roelofsen's (2013) perception study on English. Mixed-effects logistic regression was used to explore the results which revealed all dialects show a main effect of both intonation and DE choice; a rise contour and use of ?am significantly increased the likelihood of dynq responses. The effect of intonation was larger than that of DE choice in all dialects. The differences between the dialects lay in the relative strength of the DE coefficients.
\end{abstract}

Keywords: Alternative questions, disjunctive yes-no questions, intonation, prosody.

\section{Introduction}

A question like Do you want coffee or tea? can be perceived differently in English: either as an altq (possible answer: Tea) or as a dynq (possible answer: No). The two readings of the string-identical utterance are perceptually disambiguated by prosodic cues: with a fall, the utterance is perceived as an altq, but with a rise, as a dynq (Pruitt, Roelofsen 2013, Dayal 2016). They also claim that placing accents on both disjuncts ' $\mathrm{X}$ ' and ' $\mathrm{Y}$ ' (in the ' $\mathrm{X}$ or $\mathrm{Y}$ ') favours an altq reading. One reason why these questions may be disambiguated by prosody in English is that there is only one DE or which is used in both question types. Modern Standard Arabic (MSA) has two DEs: Pam in altqs and Paw in dynqs (Fakih, 2012). Colloquial Arabic dialects also display two DEs - willa and Paw - which vary in their mapping to English or. To replicate Pruitt \& Roelofsen's study in Arabic, therefore, one must first decide which DE should be used in such a perception study.

A review of corpus data and prior literature suggests that some Arabic dialects might be English-like in employing one DE in both altqs and dynqs (e.g. EA) while other dialects might be MSA-like in using two DEs, one for altqs and one for dynqs (e.g. KA). Previous research (Bani Younes, Hellmuth 2020) has found that intonation (rise) and DE choice (?aw) both favour a dynq reading in JA though both DEs are accepted in both question types, placing JA in the middle of the two preferences between English and MSA. Having established the disambiguating cues, how similar or different are the three dialects in these cues? And what is the relative contribution of each of the two

ExLing 2020: Proceedings of $11^{\text {th }}$ International Conference of Experimental Linguistics, 12-14 October 2020, Athens, Greece 
cues in the disambiguation? The present study, thus, adapts Pruitt \& Roelofsen's design for JA, EA, and KA, using the two cues: intonation (rise/fall) vs. DE choice (willa/Paw). Figure 1 below shows the same utterance produced with both contour shapes and DEs.
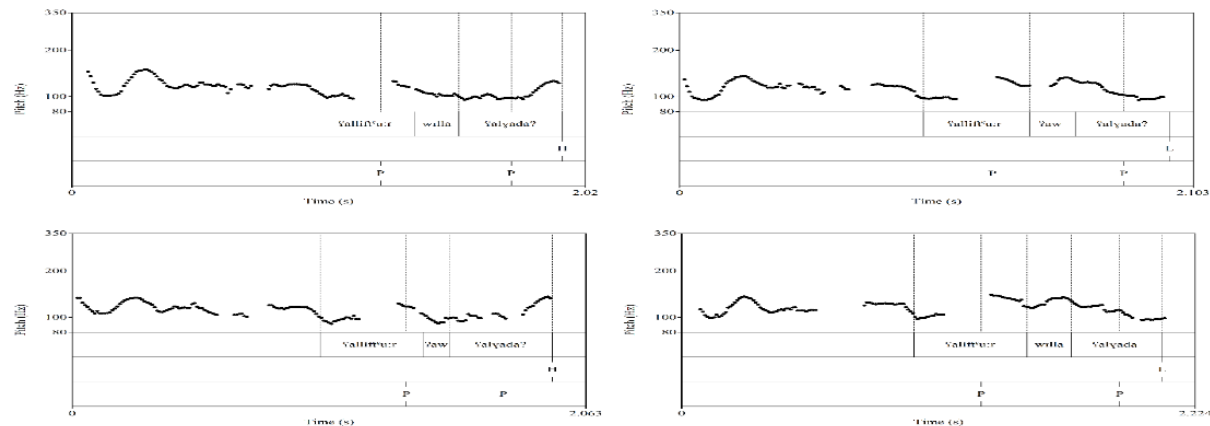

Figure 1. Contour shape vs. DE choice of Iljom Sazmatak Paia Salift' u:r Paw/willa Salyada? 'Did Aya invite you to breakfast or lunch (to have breakfast or lunch)?'

\section{Methodology}

There were $74 \mathrm{JA}, 52 \mathrm{EA}$, and $70 \mathrm{KA}$ listeners (mean age 36; range 18-53). 24 lexically-distinct target sentences and 36 fillers were recorded. The task was a two-alternative forced choice between an altq and a dynq paraphrase of the question they heard. Each target utterance was recorded in four conditions (see Figure 1 above), yielding 96 target tokens; 24 unique tokens were presented in each of four blocks in Qualtrics, in a Latin-Square design. The four conditions in each block are: 6 Paw +rise (2r), 6 Paw fall (2f), 6 willatrise (wr), and 6 willa + fall (wf) tokens. Participants were sent the experiment link and were asked to listen to the recordings via their own laptop or mobile phone in a quiet room, free from noise, using ear/headphones. After they clicked on the link, participants were randomly assigned to one of the four blocks containing 60 tokens ( 24 unique target utterances and 36 fillers). Two paraphrases appeared below each recording, so participants ticked one of them. Paraphrases were randomly ordered for each participant within each trial, and trials were also randomly ordered. After listening to each token, they selected the paraphrase that they thought was the best equivalent to what they had heard.

\section{Results and discussion}

Table 1 presents the results of the three separate GLMM models (one per dialect: glmer(resp_numeric $\sim$ intonation*DE + gender + age +Education $+(1$ + intonation $\mid$ listener $)+(1 \mid$ stimulus $)$, data $=$ data2, family $=$ binomial, control $=$ glmerControl $($ optimizer $=$ "bobyqa"))). Dynqs were coded as 1 while altqs as 0 . Figure 2 shows the spread of values across participants for counts of tokens interpreted as dynqs, by dialect. 

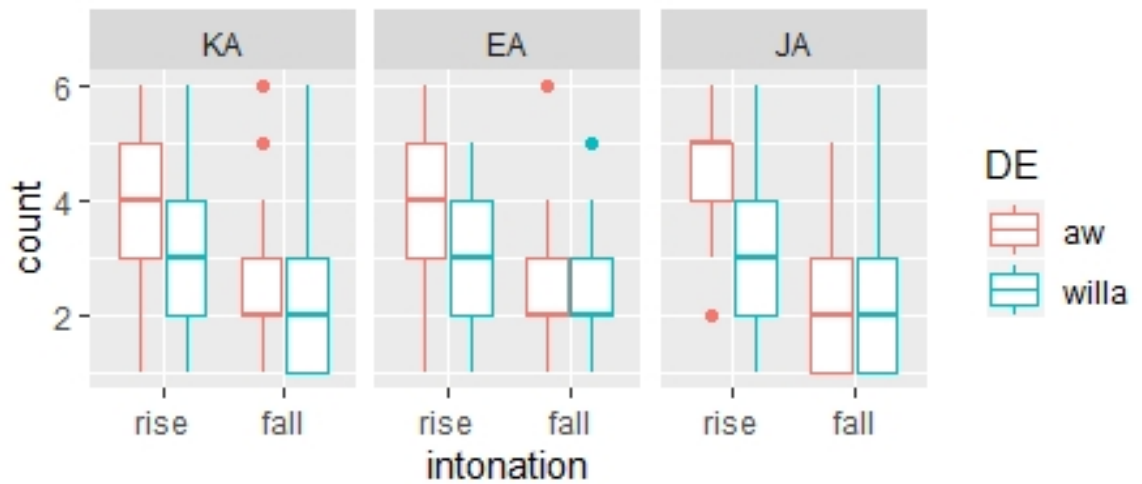

Figure 2. Median and interquartile range for count of dynq responses across participants for each dialect.

\begin{tabular}{lclcl}
\hline Fixed effects & Estimate & SE & $\mathrm{z}$ value & $\mathrm{p}$-value \\
\hline Intercept & -0.53423 & 0.52680 & -1.014 & 0.311 \\
intonation1 & 0.95554 & 0.11590 & 8.244 & $2 \mathrm{e}-16 \quad * * *$ \\
DE1 & 0.34167 & 0.08524 & 4.009 & $6.11 \mathrm{e}-05$ *** \\
intonation1:DE1 & 0.12901 & 0.08517 & 1.515 & 0.130 \\
$\quad$ Intercept & -0.2955836 & 0.4127871 & -0.716 & 0.4739 \\
intonation1 & 0.4677226 & 0.1001999 & 4.668 & $3.04 \mathrm{e}-06$ *** \\
DE1 & 0.3723088 & 0.0872576 & 4.267 & $1.98 \mathrm{e}-05$ *** \\
intonation1:DE1 & 0.1470266 & 0.0871239 & 1.688 & 0.0915. \\
Intercept & -0.225396 & 0.436883 & -0.516 & 0.6059 \\
intonation1 & 0.602045 & 0.089545 & 6.723 & $1.78 \mathrm{e}-11$ *** \\
DE1 & 0.147364 & 0.066593 & 2.213 & $0.0269 \quad *$ \\
$\quad$ intonation1:DE1 & 0.065525 & 0.066591 & 0.984 & 0.3251 \\
\hline
\end{tabular}

Table 1. Estimates of coefficients in JA, EA, and KA.

The control variables (gender, age, education) shown in the model syntax above were not included in the table as they did not reach significance. There are main effects of intonation and of DE choice in each dialect, suggesting that both cues are important in all three dialects. However, the effect size of DE choice compared with intonation is subtly different across the three dialects. JA, $\mathrm{EA}$, and $\mathrm{KA}$ are thus similar in that both intonation and DE choice significantly influence the likelihood of dynq responses. Likewise, it seems that intonation was more important than DE choice in increasing the likelihood of dynq responses in the three dialects. This matches the results of prior studies on the role of intonation in characterising the realisation of altqs and yes-no questions (ynqs) in JA, EA, and KA (e.g., Al-Khalifa 1984, Rammuny 1989, Al Amayreh 1991, Alharbi 1991, Hellmuth 2018, Winans, 2019).

The findings also corroborate the fact that the contour shape of dyngs is the same as that of ynqs. In other words, the fact that tokens with a rise 
significantly favoured dynq responses, over altq responses, is also in line with Winans' (2019) claim that ?aw-dynqs have the same contour as ynqs in EA; she explained this similarity by the fact that both are ynqs. This similarity in the contour of dynqs and ynqs might explain listeners' tendency to interpret risetokens more as dyngs in JA, EA, and KA. For this reason, interpreting tokens with a rise as dynqs is unsurprising.

The results show slight differences between the dialects. The coefficient estimates for intonation and DE in EA are similar in size, suggesting that the relative importance of $\mathrm{DE}$ choice in $\mathrm{EA}$ was the strongest across the three dialects, although intonation still plays the main role in the disambiguation. JA and KA were similar in having DE coefficient values which are much lower than those of intonation, but the effect of DE choice in KA was still smaller than that of DE choice in JA, making KA different from JA in this respect, too.

In conclusion, the findings are both similar to and different from Pruitt and Roelofsen's (2013) study on English, taking into account the idiosyncratic cues of each language. Pruitt and Roelofsen's findings suggest that two cues (intonation vs. accent distribution), both prosodic, were significant in disambiguating altqs and dynqs. They also show that intonation played the primary role while accent distribution played a supporting role. This paper adds a lexical cue (DE choice) and finds that both cues in Arabic, which are prosodic and lexical, are also needed, though the primary role is again associated with intonation and the secondary role with DE choice.

\section{References}

Al Amayreh, M.M.K. 1991. A study of the basic intonational patterns in Standard and Jordanian Arabic (Master's thesis). University of Jordan. Amman, Jordan.

Alharbi, L.M. 1991. Formal analysis of intonation: The case of the Kuwaiti Dialect of Arabic. Ph.D. Dissertation. Heriot-Watt University, UK.

Al-Khalifa, F.E. 1984. An intonational study of Kuwaiti Bedouin Arabic. Ph.D. Dissertation. University of London, UK.

Bani Younes, M., Hellmuth, S. 2020. The role of prosody in disambiguation of disjunctive questions in Jordanian Arabic. Paper presented at Arabic Linguistics Forum Conference, University of Leeds.

Dayal, V. 2016. Questions. Oxford University Press.

Fakih, A. H. 2012. The syntax of questions in Modern Standard Arabic: A minimalist perspective. Germany: LAMBERT Academic Publishing.

Hellmuth, S. 2018. Variation in polar interrogative contours within and between Arabic dialects. Proceedings of Speech Prosody. Poznań, Poland, 13-16 June.

Pruitt, K., Roelofsen, F. 2013. The interpretation of prosody in disjunctive questions. Linguistic Inquiry, 44(4), 632-650.

Rammuny, R. 1989. Instrumental and auditory analysis of Colloquial Jordanian Arabic, an update. Journal of Arabic Linguistics (20) 23-42.

Winans, L. 2019. Disjunction and alternatives in Egyptian Arabic In K. von Heusinger et al. (Eds.), Questions in Discourse (pp. 237-285). Leiden, The Netherlands: Brill. 